\title{
Risk factors of contrast-induced nephropathy in patients with STEMI and pump failure undergoing percutaneous coronary intervention
}

\author{
HONGWU CHEN ${ }^{1,2^{*}}$, XIAOFAN YU ${ }^{1,2^{*}}$ and LIKUN MA ${ }^{1,2}$ \\ ${ }^{1}$ Department of Cardiology, Division of Life Sciences and Medicine, \\ The First Affiliated Hospital of the University of Science and Technology of China (USTC), USTC; \\ ${ }^{2}$ Anhui Institute of Cardiovascular Diseases, Hefei, Anhui 230001, P.R. China
}

Received May 14, 2020; Accepted October 30, 2020

DOI: $10.3892 /$ etm.2020.9572

\begin{abstract}
Risk factors associated with the development of contrast-induced nephropathy (CIN) remain poorly defined in patients with ST-elevation myocardial infarction (STEMI) undergoing percutaneous coronary intervention (PCI). The present study was designed to assess the association between the Killip grade and the development of CIN in patients with STEMI and pump failure undergoing PCI. Data were retrospectively collected from the records of patients with STEMI and pump failure from the Chinese Society of Cardiology and American Heart Association database. A total of 7,471 patients were analyzed, including 5,521 patients with Killip grade II, 878 with Killip III and 1,072 with Killip IV pump failure. Patients were classified into two groups: Those undergoing primary PCI (PPCI; $n=5,063$ ) and those undergoing elective PCI (EPCI; $n=2,408)$. Patients in the PPCI group had higher cardiac arrest rates, lower blood pressure and higher cholesterol levels as compared to the EPCI group. There was a statistically significant difference in the rates of CIN with Killip II pump failure in the PPCI group as compared to the EPCI group, but not in those with Killip III and VI pump failure. Logistic regression analysis indicated that the Killip classification is a risk predictor for post-PCI CIN. The present results indicated a positive association between the Killip grade and post-PCI CIN in patients with STEMI and pump failure. In addition, patients with STEMI and Killip grade II pump failure were at
\end{abstract}

Correspondence to: Dr Likun Ma, Department of Cardiology, Division of Life Sciences and Medicine, The First Affiliated Hospital of the University of Science and Technology of China (USTC), USTC, 17 Lujiang Road, Hefei, Anhui 230001, P.R. China E-mail:1kma119@163.com

*Contributed equally

Key words: ST-elevation myocardial infarction, pump failure, primary percutaneous coronary intervention, contrast-induced nephropathy, Killip grade a higher risk of PCI after PPCI as compared to EPCI. Further prospective studies are required to confirm the present results.

\section{Introduction}

Percutaneous coronary intervention (PCI) is an effective treatment for the management of patients with ST-elevation myocardial infarction (STEMI) (1). However, PCI is not bereft of complications. Clinical studies have demonstrated that PCI treatment may be associated with the development of contrast-induced nephropathy (CIN) (2), due to either contrast anaphylaxis or renal hypoperfusion $(3,4)$. The development of CIN is associated with poor patient-associated outcomes, including prolonged hospital stay and increased mortality $(5,6)$. Furthermore, the risk of CIN with PCI is higher in patients with STEMI as compared to other patients requiring contrast administration. It was also indicated that no therapy is able to effectively attenuate CIN-related symptoms (7). Thus, there is a need to understand the risk factors associated with CIN in patients with STEMI undergoing PCI.

Studies have indicated that pump failure is an important risk factor for renal injury in patients with STEMI $(8,9)$. Due to decreased cardiac function, patients with STEMI and pump failure are prone to develop CIN after PCI (10). Furthermore, PCI has also been characterized as a particular risk factor for the development of CIN, probably due to the urgency of primary PCI (PPCI) to prevent pump failure or congestive heart failure (11). To reduce CIN, clinicians have used drug-based preventive strategies prior to PCI contrast administration and results have indicated a renal benefit for patients with STEMI, including improved renal function and decreased CIN event rates (12). However, such preventive therapies are frequently used in patients undergoing elective PCI (EPCI) rather than in patients undergoing PPCI (13). Furthermore, the CIN risk factors in patients with STEMI and pump failure have remained currently elusive and it should be clarified whether there are any differences in the risk between PPCI and EPCI.

Improving Care for Cardiovascular Disease in China (CCC) is a collaborative project between the American Heart Association and the Chinese Society of Cardiology (14). It is an ongoing registry and quality improvement project 
focusing on the quality of care for patients with acute coronary syndrome (ACS). The present retrospective study (based on this CCC-ACS project) on patients with STEMI and pump failure undergoing either PPCI or EPCI aimed to explore clinical risk factors for CIN. The aim was to clarify pump failure-associated risks based on Killip classes for patients with STEMI and pump failure undergoing PCI.

\section{Materials and methods}

Patients. Data from 40,343 patients with STEMI who had undergone PCI (EPCI or PPCI) within the CCC-ACS project were retrospectively collected (14). Ethical approval was provided by the institutional review board of the First Affiliated Hospital of the University of Science and Technology of China (USTC; Hefei, China).

For the study, STEMI was defined as per the 2010 guidelines issued by the Chinese Society of Cardiology (15). Patients fulfilling the following criteria were included in the study: i) Presenting within $12 \mathrm{~h}$ of the onset of symptoms (typical chest pain lasting for $>30 \mathrm{~min}$ ); ii) presenting with ST-segment elevation $\geq 1 \mathrm{~mm}$ in at least two consecutive leads; and iii) treated with stent deployment.

Exclusion criteria of the study were as follows: i) Patients who did not undergo stent deployment and those who had undergone surgical revascularization procedures or biodegradable stent implantation; ii) patients who had serum creatinine levels $>2.0 \mathrm{mg} / \mathrm{dl}$; iii) patients with post-procedural thrombolysis in myocardial infarction flow grade; iv) patients with pump failure Killip I class; and v) patients on dialysis or with end-stage kidney disease.

Data collection. The web-based data collection platform managed by each participating hospital was used for the collection of data. For creatinine measurements, reports of biochemical laboratory analyses for blood samples taken on admission and after the first $72 \mathrm{~h}$ of hospitalization were used. CIN was defined as an increase in serum creatinine by $0.5 \mathrm{mg} / \mathrm{dl}$ or $25 \%$ within $72 \mathrm{~h}$ of PCI (13).

For other variables (except for lipid profiles and glucose levels), reports of admission blood samples were used. For lipid profiles, results were obtained from fasting blood samples collected within the first $24 \mathrm{~h}$ of hospitalization. Total cholesterol, high-density lipoprotein cholesterol and triglyceride levels were measured enzymatically (Architec c-Systems; Abbott Laboratories) and low-density lipoprotein cholesterol levels were calculated from these lipid parameters using the Friedewald formula. Complete blood count analyses were performed using standard clinical laboratory methods (Coulter LH 780 Hematology Analyzer; Beckman Coulter) and hemoglobin and glycated hemoglobin (HbA1c) were determined.

Coronary angiography data and Killip grading. Angiographic PPCI or EPCI data obtained directly from the cardiac catheterization laboratory records were examined by two independent researchers (HC and XY), who were blinded to the patient data. When there was a difference of opinion, we discussed together and came to a unanimous conclusion. The Killip classification $(16,17)$ was used for grading pump failure as follows: i) Killip I: Absence of heart failure symptoms but increased pulmonary capillary wedge pressure; ii) Killip II: Mild to moderate heart failure, lung rales in $<50 \%$ of the two lungs. X-ray characteristics of pulmonary venous plethora; iii) Killip III: Severe heart failure with acute pulmonary edema. Lung rales present in $>50 \%$ of the two lungs; iv) Killip IV: Cardiogenic shock.

Statistical analysis. All continuous variables were expressed as the mean \pm standard deviation and all categorical variables as $n(\%)$. The assumption of a normal distribution was tested for continuous variables using a Kolmogorov-Smirnov analysis. Differences in continuous variables between groups were assessed using Student's t-test or the Mann-Whitney U test and categorical variables were compared using Pearson's Chi-square test or Fisher's exact test. Logistic regression analyses were performed to investigate the univariable and multivariable predictors with $95 \%$ CI. $\mathrm{P}<0.05$ was considered to indicate statistical significance. Variables with $\mathrm{P}<0.10$ and the other variables that have been reported to be associated with contrast-induced nephropathy in patients with STEMI and pump failure were included in the univariate analysis of the multivariable logistic regression model. Included variables were age, male sex, Killip grade, hypertension, primary PCI, chronic heart failure, chronic kidney failure, smoking, old myocardial infarction, post-PCI, post-CABG, diabetes mellitus, family history of CHD, hyperlipemia. All statistical analyses were performed using the Statistical Package for Social Sciences software (SPSS 20.0 for Windows; IBM Corp.).

\section{Results}

Patients. A total of 7,471 patients fulfilled the inclusion criteria for the analysis (5,521 with Killip grade II, 878 with Killip III and 1,072 with Killip IV). Patients were further divided into two groups, with 5,063 patients in the PPCI group and 2,408 in the EPCI group (Fig. 1). The mean age of the patients in the PPCI and EPCI group was $63.6 \pm 12.5$ and $64 \pm 11.6$ years, respectively. A total of $78.2 \%$ of patients in the PPCI group and $76.2 \%$ in the EPCI group were male.

Baseline variables. Details of the baseline characteristics of the study groups are presented in Table I. There were no statistically significant differences in terms of sex, age, body mass index, hypertension, hyperlipemia, diabetes mellitus, ongoing myocardial infarction, post-PCI, post-coronary artery bypass graft, heart rate, triglyceride and glycated hemoglobin between the two groups. The percentage of patients with family history of congestive heart disease and that of smokers was significantly higher in the PPCI group, while the percentage of patients with chronic heart failure was significantly higher in the EPCI group $(\mathrm{P}<0.05)$. Comparison of cardiac arrest rates and related variables indicated that the incidence of cardiac arrest was higher in the PPCI group (5.3\%) compared to the EPCI group (3.2\%; $\mathrm{P}<0.001$; Table I). Furthermore, the mean systolic $(\mathrm{P}<0.001)$ and diastolic $(\mathrm{P}<0.01)$ blood pressures were significantly lower in the PPCI group as compared with that in the EPCI group, providing a mechanism for the increased cardiac arrest rates. 
Table I. Comparison of baseline data between the PPCI and EPCI groups.

\begin{tabular}{|c|c|c|c|c|}
\hline Variable & Total $(n=7,471)$ & PPCI $(n=5,063)$ & EPCI $(n=2,408)$ & $\mathrm{P}$-value \\
\hline Male sex & $5,792(77.5)$ & $3,958(78.2)$ & $1,834(76.2)$ & 0.051 \\
\hline Age (years) & $63.7 \pm 12.2$ & $63.6 \pm 12.5$ & $64.0 \pm 11.6$ & 0.233 \\
\hline BMI & $24.2 \pm 3.3$ & $24.2 \pm 3.2$ & $24.2 \pm 3.6$ & 0.699 \\
\hline Smoking & $3,437(46.0)$ & $2,372(46.8)$ & $1,065(44.2)$ & 0.034 \\
\hline Hypertension & $3,681(49.3)$ & $2,486(49.1)$ & $1,195(49.6)$ & 0.671 \\
\hline Hyperlipemia & $424 \quad(5.7)$ & $284(5.6)$ & $140(5.8)$ & 0.721 \\
\hline Diabetes mellitus & $1,592(21.3)$ & $1,050(20.7)$ & $542(22.5)$ & 0.081 \\
\hline Family history of CHD & $151(2.0)$ & $116(2.3)$ & $35(1.5)$ & 0.016 \\
\hline Chronic heart failure & $91(1.2)$ & $50(1.0)$ & $41(1.7)$ & 0.008 \\
\hline Chronic kidney failure & $71(1.0)$ & $48(0.9)$ & $23(1.0)$ & 0.976 \\
\hline OMI & $411(5.5)$ & $271(5.4)$ & $140(5.8)$ & 0.414 \\
\hline Post-PCI & $352(4.7)$ & 247 (4.9) & $105(4.4)$ & 0.323 \\
\hline Post-CABG & $18(0.2)$ & $13(0.3)$ & $5(0.2)$ & 0.686 \\
\hline HR (bpm) & $80.1 \pm 18.6$ & $80.4 \pm 19.2$ & $79.6 \pm 17.2$ & 0.074 \\
\hline HbA1c (\%) & $6.71 \pm 1.70$ & $6.67 \pm 1.70$ & $6.80 \pm 1.70$ & 0.077 \\
\hline TG (mmol/l) & $1.66 \pm 1.19$ & $1.66 \pm 1.23$ & $1.66 \pm 1.12$ & 0.903 \\
\hline Cardiac arrest & $344(4.6)$ & $266(5.3)$ & $78(3.2)$ & $<0.001$ \\
\hline DBP (mmHg) & $75.4 \pm 15.5$ & $75.1 \pm 16.1$ & $76.1 \pm 14.1$ & 0.009 \\
\hline $\mathrm{SBP}(\mathrm{mmHg})$ & $123.5 \pm 24.9$ & $122.7 \pm 25.8$ & $125.2 \pm 22.9$ & $<0.001$ \\
\hline $\mathrm{TC}(\mathrm{mmol} / \mathrm{l})$ & $4.46 \pm 1.25$ & $4.54 \pm 1.26$ & $4.28 \pm 1.23$ & $<0.001$ \\
\hline LDL-C (mmol/l) & $1.11 \pm 0.43$ & $2.87 \pm 1.00$ & $2.67 \pm 0.90$ & $<0.001$ \\
\hline HDL-C (mmol/l) & $2.81 \pm 0.97$ & $1.13 \pm 0.45$ & $1.07 \pm 0.39$ & $<0.001$ \\
\hline
\end{tabular}

Values are expressed as the mean \pm standard deviation or $\mathrm{n}(\%)$. PPCI, primary PCI; EPCI, elective PCI; BMI, body mass index; CHD, congestive heart disease; OMI, old myocardial infarction; PCI, percutaneous coronary intervention; CABG, coronary artery bypass graft; DBP, diastolic blood pressure; HR, heart rate; bpm; beats per minute; TG, triglyceride; HbA1c, glycated hemoglobin; TC, total cholesterol; SBP, systolic blood pressure; LDL-C, low-density lipoprotein cholesterol; HDL-C, high-density lipoprotein cholesterol.

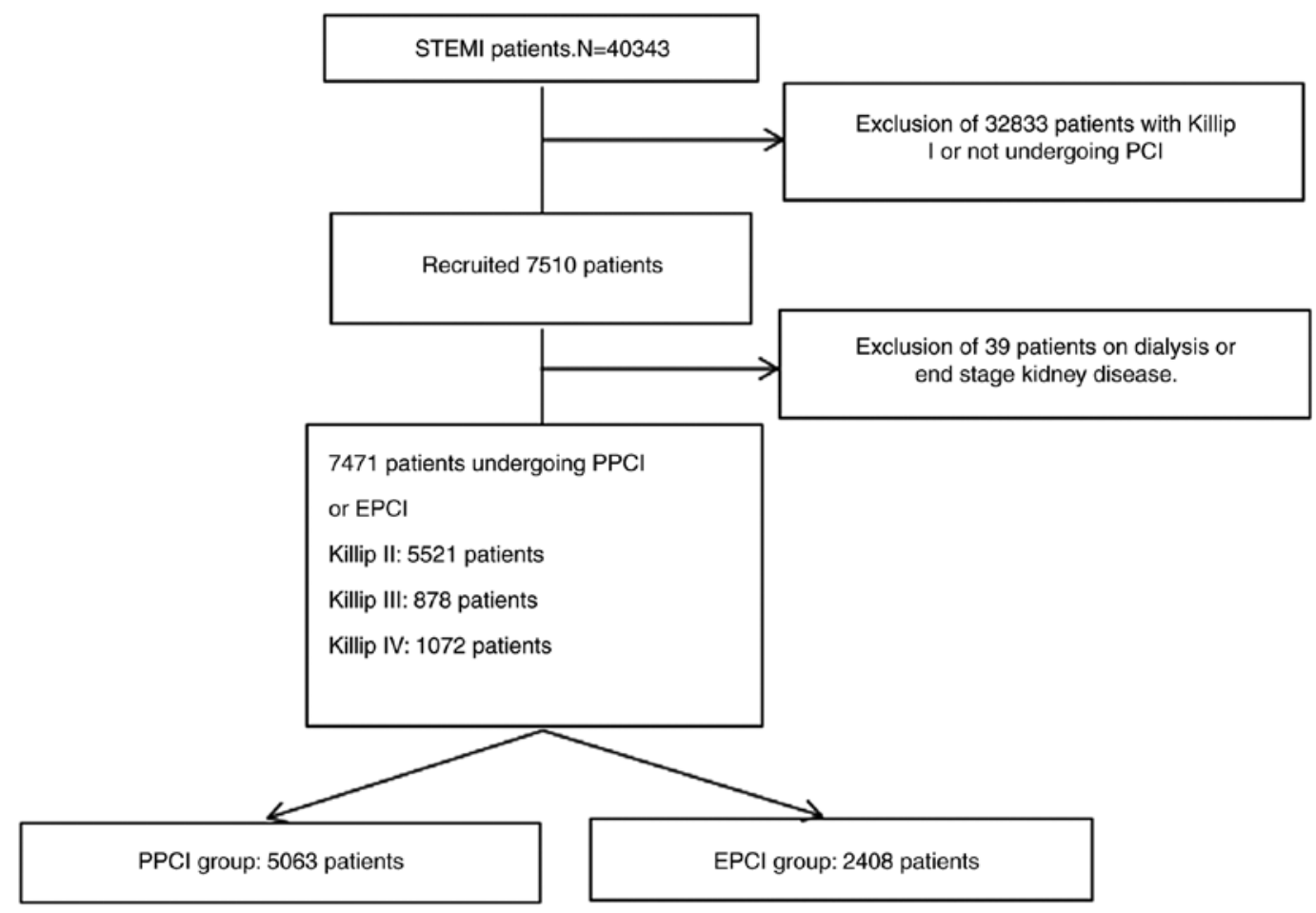

Figure 1. Flow diagram for the present retrospective study. PPCI, primary PCI; EPCI, elective PCI; PCI, percutaneous coronary intervention; STEMI, ST-elevation myocardial infarction. 
Table II. Comparison of culprit artery and related infarction area.

\begin{tabular}{llll}
\hline Variable & Total $(\mathrm{n}=7,471)$ & PPCI $(\mathrm{n}=5,063)$ & EPCI $(\mathrm{n}=2,408)$ \\
\hline Culprit artery & & & P-value \\
LM & $130(1.7)$ & $102(2.0)$ & $28(1.2)$ \\
LAD & $4,379(58.6)$ & $2,934(57.9)$ & $1,445(60.0)$ \\
LCX & $850(11.4)$ & $552(10.9)$ & $298(12.4)$ \\
RCA & $2,030(27.2)$ & $1,427(28.2)$ & $603(25.0)$ \\
SVG & $19(0.3)$ & $10(0.2)$ & $9(0.4)$ \\
Others & $63(0.8)$ & $38(0.8)$ & $25(1.0)$ \\
Simultaneous treatment of & $616(8.2)$ & $333(6.6)$ & $283(11.8)$ \\
non-culprit artery & $4,509(60.4)$ & $3,036(60.0)$ & $1,473(61.2)$ \\
Anterior myocardial infarction & & & 0.001 \\
\hline
\end{tabular}

Values are expressed as the mean \pm standard deviation or $\mathrm{n}(\%)$. PPCI, primary PCI; EPCI, elective PCI; PCI, percutaneous coronary intervention; LM, left main coronary artery; LAD, left anterior descending artery; LCX, left circumflex artery; RCA, right circumflex artery; SVG, saphenous venous graft.

Table III. Comparison of CIN between the PPCI and EPCI groups.

\begin{tabular}{lccr}
\hline Variable & Total $(\mathrm{n}=7,471)$ & PPCI $(\mathrm{n}=5,063)$ & EPCI $(\mathrm{n}=2,408)$ \\
\hline Pre-PCI Cr (mmol/l) & $86.7 \pm 37.4$ & $86.4 \pm 37.3$ & $87.2 \pm 37.7$ \\
Pre-PCI eGFR (ml/min) & $96.78 \pm 50.05$ & $97.12 \pm 49.97$ & $96.08 \pm 50.23$ \\
Maximum Cr (mmol/l) & $105.60 \pm 63.99$ & $106.88 \pm 67.82$ & $102.92 \pm 55.95$ \\
CIN incidence & $1,212(16.2)$ & $860(17.0)$ & $352(14.6)$ \\
Killip grade & & & 0.403 \\
II & $824(14.9)$ & $580(15.7)$ & $244(13.4)$ \\
III & $175(19.9)$ & $112(21.3)$ & $63(17.9)$ \\
IV & $213(19.9)$ & $168(20.2)$ & $45(18.8)$ \\
\hline
\end{tabular}

Values are expressed as the mean \pm standard deviation or $\mathrm{n}(\%)$. PPCI, primary PCI; EPCI, elective PCI; PCI, percutaneous coronary intervention; $\mathrm{Cr}$, creatinine; eGFR, estimated glomerular filtration rate; CIN, contrast-induced nephropathy.

In addition, cholesterol levels related to cardiac arrest onset were also higher in the PPCI group $(\mathrm{P}<0.001)$. The above results demonstrated that the patients in the PPCI group, as expected, exhibited emergency features in line with the emergency treatment needed in this group.

Culprit arteries and myocardial injury during PCI treatment. Data on the frequencies of culprit arteries and myocardial injury were analyzed from the patients' records. The culprit artery distributions varied depending on the group. The proportions of left main coronary artery and right circumflex artery were both higher in the PPCI group, while those of left anterior descending, left circumflex artery and saphenous venous graft lesions were smaller than those in the EPCI group $(\mathrm{P}<0.05$; Table II). In addition, the incidence of anterior myocardial infarction in the PPCI group (60\%) was significantly lower than that in the EPCI group (61.2\%; P<0.001; Table II), while simultaneous treatment of non-culprit arteries was less common in the PPCI group (6.6\%) than in the EPCI group (11.8\%; $\mathrm{P}<0.001$; Table II).
Incidence of CIN. Clinical parameters of renal factors, including pre-PCI creatinine and pre-PCI estimated glomerular filtration rate, were not significantly different between the PPCI and EPCI groups (P>0.05; Table III). After PCI treatment, the patients in the PPCI group had significantly higher post-PCI maximum creatinine levels $(\mathrm{P}<0.05)$ as compared to the patients in the EPCI group. In addition, the incidence of CIN was significantly higher in the PPCI group (17\%) as compared to the EPCI group $(\mathrm{P}<0.05)$. Further subgroup analyses based on pump failure demonstrated that only patients with Killip II pump failure had a statistically significant increase in the incidence of CIN in the PPCI group as compared to the EPCI group $(\mathrm{P}<0.05)$. No such difference was noted in patients with Killip III and IV pump failure.

Logistic regression analysis indicated that age, sex, the Killip classification, hypertension, PPCI vs. EPCI, presence of chronic heart failure and chronic renal failure were the major independent variables predicting post-PCI CIN (Table IV). Specifically, Killip classification was positively associated with an increased risk of CIN after PCI with an odds ratio of 1.160 (95\% CI, 1.069-1.259). 
Table IV. Logistic regression analysis for contrast-induced nephropathy.

\begin{tabular}{|c|c|c|c|c|}
\hline Variable & Unadjusted OR (95\% CI) & P-value & Adjusted OR (95\% CI) & P-value \\
\hline Age (per unit increase in years) & $1.024(1.019-1.030)$ & $<0.001$ & $1.019(1.013-1.025)$ & $<0.001$ \\
\hline Sex (male vs. female) & $0.651(0.567-0.747)$ & $<0.001$ & $0.805(0.689-0.941)$ & 0.006 \\
\hline Killip class (per unit increase in grade) & $1.214(1.121-1.315)$ & $<0.001$ & $1.160(1.069-1.259)$ & $<0.001$ \\
\hline Hypertension (present vs. absent) & $0.747(0.660-0.845)$ & $<0.001$ & $0.840(0.739-0.955)$ & 0.008 \\
\hline Type of PCI (PPCI vs. EPCI) & $1.195(1.044-1.368)$ & 0.010 & $1.198(1.044-1.373)$ & 0.010 \\
\hline Chronic heart failure (present vs. absent) & $0.453(0.288-0.714)$ & 0.001 & $0.579(0.357-0.939)$ & 0.027 \\
\hline Chronic kidney failure (present vs. absent) & $0.276(0.171-0.444)$ & $<0.001$ & $0.335(0.206-0.547)$ & $<0.001$ \\
\hline Smoking (present vs. absent) & $1.377(1.215-1.561)$ & $<0.001$ & $1.093(0.924-1.294)$ & 0.300 \\
\hline OMI (present vs. absent) & $1.013(0.773-1.328)$ & 0.926 & $1.148(0.720-1.828)$ & 0.562 \\
\hline Post-PCI (present vs. absent) & $1.002(0.750-1.340)$ & 0.988 & $1.189(0.725-1.951)$ & 0.493 \\
\hline Post-CABG (present vs. absent) & $0.677(0.222-2.060)$ & 0.492 & $1.022(0.216-4.84)$ & 0.978 \\
\hline Diabetes mellitus (present vs. absent) & $0.813(0.703-0.939)$ & 0.005 & $0.951(0.789-1.145)$ & 0.593 \\
\hline Family history of CHD (present vs. absent) & $0.975(0.632-1.505)$ & 0.911 & $1.050(0.604-1.828)$ & 0.862 \\
\hline Hyperlipemia (present vs. absent) & $0.944(0.727-1.225)$ & 0.663 & $0.816(0.596-1.117)$ & 0.204 \\
\hline
\end{tabular}

Adjusted variables: Age, male sex, Killip grade, hypertension, primary PCI, chronic heart failure, chronic kidney failure, smoking, old myocardial infarction, post-PCI, post-CABG, diabetes mellitus, family history of CHD and hyperlipemia. PPCI, primary PCI; EPCI, elective PCI; PCI, percutaneous coronary intervention; OR, odds ratio; OMI, old myocardial infarction; CABG, coronary artery bypass grafting; CHD, coronary heart disease.

\section{Discussion}

The present results indicated that the incidence of CIN in patients with STEMI undergoing PPCI with Killip grade II pump failure is higher as compared to those undergoing EPCI. This difference is not apparent in other Killip classes. In addition, the present analysis demonstrated that the Killip classification is positively associated with post-PCI CIN onset for patients with STEMI and pump failure.

PPCI is the gold standard treatment for patients with STEMI, particularly for those with pump failure. On the other hand, pump failure is an important predictor for CIN (18). For efficient management of patients with pump failure, coronary artery disease, acute coronary syndrome or STEMI, risk factors for CIN must be recognized early so that appropriate management protocols are followed (19). The present study was focused on the subgroup of patients with STEMI and pump failure, which is known to be significantly affected by CIN (18). Studies have demonstrated that oxidative stress, inflammation and renal ischemia/reperfusion are the major potential pathophysiological mechanisms of CIN, while perfusion markers (including the Killip grade) have been indicated to predict the risk of CIN $(20,21)$. On the other hand, impaired renal function is a risk factor for cardiovascular diseases and pre-admission renal parameters or worsening of renal function during hospitalization have been reported to indicate the risk for adverse events in patients with STEMI $(22,23)$.

Similar to those of a previous study (24), the present results indicated a positive correlation between the development of CIN and PPCI treatment. The present retrospective analysis also demonstrated a higher incidence of cardiac arrest events, lower SBP values and higher cholesterol levels in the PPCI group as compared with those in the EPCI group. These results are consistent with those of previous studies focusing on patients with PPCI $(25,26)$. In addition, the incidence of anterior myocardial infarction in the PPCI group was significantly lower than that in the EPCI group, while simultaneous treatment of non-culprit arteries was less frequent in the PPCI group. Of note, the simultaneous treatment of non-culprit arteries has been reported as a protective factor against CIN onset (27), which may partially explain the difference in CIN incidence rates between the groups of the present study. In the present study, patients in the PPCI group had a higher incidence of CIN than those in the EPCI group. Similar results have been reported previously (28).

The effects of pump failure on CIN remain to be fully elucidated. More specifically, the association between the Killip grade and the development of CIN in patients with STEMI after PPCI has remained to be explored. The regression analysis of the present study demonstrated that failing pump function was significantly associated with an increased risk of CIN. Furthermore, patients with Killip grade II in the PPCI group had a significantly higher incidence of CIN as compared with those in the EPCI group. However, there was no significant difference in the incidence of PCI among the Killip III and IV subgroups, despite the greater severity of pump failure in these patients. The lack of significance in the incidence of CIN between the PPCI and EPCI patients with Killip III or IV with pre-PCI renal hypoperfusion may be attributed to their prior treatment for heart failure (before PPCI), thereby restoring their renal reperfusion and diminishing renal injuries after contrast administration. Thus, patients with Killip II without severe renal dysfunction, who are not pretreated before the PPCI, may develop ischemia-reperfusion renal injury more frequently as compared to patients with more severe pump failure. 
As for contrast dosage and type, previous studies have demonstrated that the contrast dosage is associated with the post-PCI CIN incidence in patients with STEMI $(29,30)$. In addition, hypotonic and hyperosmotic contrasts display similar risk profiles for CIN (31). Therefore, the influence of contrast media on CIN was not analyzed in the present study.

The limitations of the present study should be mentioned. The retrospective nature of the analysis prevents us from drawing a causal association between the presence of CIN and the development of pump failure. Only randomized clinical trials are able to firmly establish such an association. In addition, the establishment of an animal model will improve our understanding of the detailed mechanisms.

In conclusion, the Killip classification was positively associated with post-PCI CIN events in patients with STEMI and pump failure. The present results also indicate that patients with Killip II grade pump failure undergoing PPCI are at a higher risk of CIN as compared to those undergoing EPCI and there is a need for adequate preventive measures to avoid the development of CIN in such individuals. Since our conclusions are derived from a retrospective cohort, there is a need for further prospective studies to confirm the present results.

\section{Acknowledgements}

Not applicable.

\section{Funding}

This study was supported by the CCC-ACS project; the Open Research Fund of Anhui Institute of Cardiovascular Diseases (grant no. KF2018007) and 'Borrow to transfer to supplement' project of Hefei independent innovation policy (grant no. J2019Y02).

\section{Availability of data and materials}

The datasets used and/or analyzed during the current study are available from the corresponding author on reasonable request.

\section{Authors' contributions}

HC, XY and LM conceived and designed the study. HC and $\mathrm{XY}$ were involved in data collection, interpretation and analysis, and in writing of the manuscript. LM was involved in the editing of the manuscript. All authors read and approved the final manuscript.

\section{Ethics approval and consent to participate}

The Ethics Committee of the First Affiliated Hospital of USTC approved this study (no. 2020-P-053). Informed consent was waived due to the retrospective nature of the study and the use of anonymized clinical data.

\section{Patient consent for publication}

Not applicable.

\section{Competing interests}

The authors declare that they have no competing interests.

\section{References}

1. Keeley EC, Boura JA and Grines CL: Primary angioplasty versus intravenous thrombolytic therapy for acute myocardial infarction: A quantitative review of 23 randomised trials. Lancet 361: 13-20, 2003.

2. Turak O, Ozcan F, Isleyen A, Tok D, Sokmen E, Buyukkaya E, Aydogdu S, Akpek M and Kaya MG: Usefulness of the neutrophil-to-lymphocyte ratio to predict bare-metal stent restenosis. Am J Cardiol 110: 1405-1410, 2012.

3. De Luca G, Dirksen MT, Spaulding C, Kelbaek H, Schalij M, Thuesen L, van der Hoeven B, Vink MA, Kaiser C, Musto C, et al; Drug-Eluting Stent in Primary Angioplasty (DESERT) Cooperation: Drug-eluting vs bare-metal stents in primary angioplasty: A pooled patient-level meta-analysis of randomized trials. Arch Intern Med 172: 611-622, 2012.

4. Jukema JW, Verschuren JJW, Ahmed TAN and Quax PHA: Restenosis after PCI. Part 1: Pathophysiology and risk factors. Nat Rev Cardiol 9: 53-62, 2011.

5. Li J-J, Ren Y, Chen K-J, Yeung AC, Xu B, Ruan XM, Yang YJ, Chen JL and Gao RL: Impact of C-reactive protein on in-stent restenosis: A meta-analysis. Tex Heart Inst J 37: 49-57, 2010.

6. Bolca O, Güngör B, Özcan KS, Karadeniz FÖ, Sungur A, Köroğlu B, Bakhshyaliyev N, Yelgeç NS, Karataş B, İpek G, et al: The neutrophil-to-lymphocyte ratio is associated with bare-metal stent restenosis in STEMI patients treated with primary PCI. Coron Artery Dis 26: 402-408, 2015.

7. AlFaleh HF, Alsuwaida AO, Ullah A, Hersi A, AlHabib KF, AlNemer K, AlSaif S, Taraben A, Kashour T, Balghith MA, et al: The prognostic impact of in-hospital worsening of renal function in patients with acute coronary syndrome. Int J Cardiol 167: 866-870, 2013

8. Amin AP, Spertus JA, Reid KJ, Lan X, Buchanan DM, Decker C and Masoudi FA: The prognostic importance of worsening renal function during an acute myocardial infarction on long-term mortality. Am Heart J 160: 1065-1071, 2010.

9. Goldberg A, Hammerman H, Petcherski S, Zdorovyak A Yalonetsky S, Kapeliovich M, Agmon Y, Markiewicz W and Aronson D: Inhospital and 1-year mortality of patients who develop worsening renal function following acute ST-elevation myocardial infarction. Am Heart J 150: 330-337, 2005.

10. Stefanini GG, Taniwaki M, Kalesan B, Räber L, Stortecky S, Pilgrim T, Onuma Y, Silber S, Serruys PW, Meier B, et al: The impact of renal impairment on long-term safety and effectiveness of drug-eluting stents. PLoS One 9: e106450, 2014.

11. Cassese S, Byrne RA, Tada T, Pinieck S, Joner M, Ibrahim T, King LA, Fusaro M, Laugwitz KL and Kastrati A: Incidence and predictors of restenosis after coronary stenting in 10004 patients with surveillance angiography. Heart 100: 153-159, 2014.

12. Best PJM, Berger PB, Davis BR, Grines CL, Sadeghi HM, Williams BA, Willerson JT, Granett JR and Holmes DR Jr; PRESTO Investigators: Impact of mild or moderate chronic kidney disease on the frequency of restenosis: Results from the PRESTO trial. J Am Coll Cardiol 44: 1786-1791, 2004.

13. Marenzi G, Lauri G, Assanelli E, Campodonico J, De Metrio M, Marana I, Grazi M, Veglia F and Bartorelli AL: Contrast-induced nephropathy in patients undergoing primary angioplasty for acute myocardial infarction. J Am Coll Cardiol 44: 1780-1785, 2004.

14. Hao Y, Liu J, Liu J, Smith SC Jr, Huo Y, Fonarow GC, Ma C, Ge J, TaubertKA, Morgan L, et al; CCC-ACS Investigators: Rationale and design of the Improving Care for Cardiovascular Disease in China (CCC) project: A national effort to prompt quality enhancement for acute coronary syndrome. Am Heart J 179: 107-115, 2016.

15. Shen W and Gao R: Chinese Society of Cardiology. Guideline for diagnosis and treatment of patients with ST-elevation myocardial infarction. China J Cardiol 38: 675-690, 2010.

16. Killip T III and Kimball JT: Treatment of myocardial infarction in a coronary care unit. A two year experience with 250 patients. Am J Cardiol 20: 457-464, 1967.

17. Khot UN, Jia G, Moliterno DJ, Lincoff AM, Khot MB, Harrington RA and Topol EJ: Prognostic importance of physical examination for heart failure in non-ST-elevation acute coronary syndromes: The enduring value of Killip classification. JAMA 290: 2174-2181, 2003. 
18. Yamaguchi J, Kasanuki H, Ishii Y, Yagi M, Nagashima M, Fujii S, Koyanagi R, Ogawa H, Hagiwara N, Haze K, et al; HIJC Investigators: Serum creatinine on admission predicts long-term mortality in acute myocardial infarction patients undergoing successful primary angioplasty: Data from the Heart Institute of Japan Acute Myocardial Infarction (HIJAMI) Registry. Circ J 71: 1354-1359, 2007.

19. Halkin A, Mehran R, Casey CW, Gordon P, Matthews R, Wilson BH, Leon MB, Russell ME, Ellis SG and Stone GW: Impact of moderate renal insufficiency on restenosis and adverse clinical events after paclitaxel-eluting and bare metal stent implantation: Results from the TAXUS-IV Trial. Am Heart J 150: 1163-1170, 2005.

20. Wang C-H, Zhang S-Y, Fang Q, Shen ZJ, Fan ZJ, Jin XF, Zeng Y, Liu ZY and Xie HZ: Renal Dysfunction and hsCRP Predict Long-term Outcomes of Percutaneous Coronary Intervention in Acute Myocardial Infarction. Am J Med Sci 349: 413-420, 2015.

21. Zhang RY, Zhu ZB, Zhang Q, Yang ZK, Hu J, Lv AK, Zhang JS and Shen WF: Impact of moderate or severe renal insufficiency on long-term outcomes in patients undergoing drug-eluting stent based coronary intervention. Int J Cardiol 136: 72-79, 2009.

22. Garg P, Charytan DM, Novack L, Cutlip DE, Popma JJ, Moses J, Leon MB, Schofer J, Breithardt G, Schampaert E, et al: Impact of moderate renal insufficiency on restenosis and adverse clinical events after sirolimus-eluting and bare metal stent implantation (from the SIRIUS trials). Am J Cardiol 106: 1436-1442, 2010.

23. Sahin I, Gungor B, Can MM, Avci II, Guler GB, Okuyan E, Biter H, Yildiz SS, Ayca B, Satilmis S, et al: Lower blood vitamin D levels are associated with an increased incidence of contrast-induced nephropathy in patients undergoing coronary angiography. Can J Cardiol 30: 428-433, 2014

24. Seyfarth M, Kastrati A, Mann JFE, Ndrepepa G, Byrne RA, Schulz S, Mehilli J and Schömig A: Prognostic value of kidney function in patients with ST-elevation and non-ST-elevation acute myocardial infarction treated with percutaneous coronary intervention. Am J Kidney Dis 54: 830-839, 2009.

25. Rubartelli P, Niccoli L, Verna E, Giachero C, Zimarino M Fontanelli A, Vassanelli C, Campolo L, Martuscelli E and Tommasini G: Stent implantation versus balloon angioplasty in chronic coronary occlusions: Results from the GISSOC trial. Gruppo Italiano di Studio sullo Stent nelle Occlusioni Coronariche. J Am Coll Cardiol 32: 90-96, 1998.
26. Sahin I, Karabulut A, Avci II, Okuyan E, Biter HI, Yildiz SS, Can MM, Gungor B, Dinckal M and Serebruany V: Contribution of platelets indices in the development of contrast-induced nephropathy. Blood Coagul Fibrinolysis 26: 246-249, 2015.

27. Börekçi A, Gür M, Türkoğlu C, Çaylı M, Selek Ş, Kaypaklı O, Uçar H, Coşkun M, Seker T, Koç M, et al: Oxidative stress and paraoxonase 1 activity predict contrast-induced nephropathy in patients with ST-segment elevation myocardial infarction undergoing primary percutaneous coronary intervention. Angiology 66: 339-345, 2015.

28. Mizuguchi Y, Shibutani H, Hashimoto S, Yamada T, Taniguchi N, Nakajima S, Hata T and Takahashi A: Examination of the appropriate timing of reperfusion therapy for recent myocardial infarction: A Japanese single-center retrospective study. Indian Heart J 70: 4-9, 2018.

29. Caspi O, Habib M, Cohen Y, Kerner A, Roguin A, Abergel E, Boulos M, Kapeliovich MR, Beyar R, Nikolsky E, et al: Acute Kidney Injury After Primary Angioplasty: Is Contrast-Induced Nephropathy the Culprit? J Am Heart Assoc 6: 6, 2017.

30. Velibey Y, Tanik O, Oz A, Guvenc TS, Kalenderoglu K, Gumusdag A, Guzelburc O, Tekkesin AI, Uzun AO, Alper AT, et al: Off-Hour Primary Percutaneous Coronary Angioplasty Does Not Affect Contrast-Induced Nephropathy in Patients With ST-Segment Elevation Myocardial Infarction. Angiology 68: 807-815, 2017.

31. Marrón B, Ruiz E, Fernández C, Almeida P, Horcajada C, Navarro F and Caramelo C: Systemic and renal effects of preventing contrast nephrotoxicity with isotonic $(0.9 \%)$ and hypotonic (0.45\%) saline. Rev Esp Cardiol 60: 1018-1025, 2007 (In Spanish).

This work is licensed under a Creative Commons Attribution-NonCommercial-NoDerivatives 4.0 International (CC BY-NC-ND 4.0) License. 